\title{
Optimal Green-advertising Incentive Model in a Closed-loop Supply Chain
}

\author{
Yang $\mathrm{Bai}^{1}$ and Byeong-Yun Chang ${ }^{1, *}$ \\ ${ }^{1}$ School of Business, Ajou University, Suwon, Republic of Korea \\ b198716y@ajou.ac.kr,*bychang@ajou.ac.kr
}

\begin{abstract}
This research investigates green-advertising incentive strategies in a dynamic multichannel closed-loop supply chain (CLSC) where a manufacturer as a leader directs both traditional retailer channels and e-tail (online) channels. We built three different greenadvertising incentive models: a non-co-op green-advertising incentive model (NGA Model,) where both of the players invest in green advertising, separately; a unilateral coop green-advertising incentive model (CGA Model), where the manufacturer supports part of the retailer's green-advertising cost incentive to increase its green-advertising investments; and a bilateral co-op green-advertising incentive model (BGA Model), where the manufacturer and retailer support part of each other's green-advertising costs incentive to increase their green-advertising investments. For each of these three models, the optimal price and green-advertising incentive decisions were obtained. The results show that the bilateral co-op green-advertising incentive model (BGA Model) is much stronger than the other two green-advertising incentive strategies in CLSC system. A manufacturer can use a bilateral co-op green-advertising incentive strategy to stimulate and influence the customers' awareness about environmental issues, promote customers' return rate of used/end-of-life products, and ultimately achieve the goals of profit maximization and sustainable development.
\end{abstract}

Keywords: closed-loop supply chain; green advertising; incentive mechanisms; equilibrium; remanufacturer

\section{Introduction}

Given the rapid development of online technology, many manufacturers (or suppliers), such as IBM, P\&G and HP, are introducing direct e-tail (online) orders to redesign their supply-chain channels [1-3]. Meanwhile, with the environmental degradation and resource shortage, more and more enterprises recognize the importance of sustainable development. As Bulmus et al., [4] reports, the consumption of remanufactured products in the U.S. grew to $\$ 43$ billion by 2011 , up from $\$ 37.3$ billion in 2009. Companies such as Ford and IBM, through reverse logistics remanufacturing of used products in the closed-loop supply chain (CLSC) to save raw materials, effectively allocate existing resources and material recycling, and ultimately achieve the goals of profit maximization and sustainable development. The CLSC is a closed-loop system that combines both forward and reverse logistics aimed at achieving the challenge of environmental, economic and social performance [5-17].

There is increased interest in research on how to effectively manage the reverse logistics in CLSC system. Lu et al. [18] and De Giovanni et al., [19] show that incentive strategies allow the decentralized CLSC to have the same effect of economical and environmental performance as centralized CLSC has. In a decentralized CLSC system, manufacturers may

Received (July 21, 2018), Review Result (September 29, 2018), Accepted (October 10, 2018)

* Corresponding Author 
use a variety of reverse logistics to collect used/end-of-life products, such as by remanufacturing directly, collecting from consumers, retailers collecting from consumers, or third-party collecting from consumers. Savaskan et al., [20] compared three different options of collecting activities. Saha et al., [21] investigated a dual CLSC coordination and compared three different models of collection types. Savaskan and Van Wassenhove [22] show us that retailer collection is the most effective for the success of a CLSC. On the other hand, the retailer is motivated to close the loop only if there is an attractive economic incentive [23-24]. De Giovanni [25-26] shows that the incentive always turns out to be more effective in the CLSC.

Therefore, how to stimulate the players to close the loop has attracted more attention from researchers. Corbett and Savaskan [27 show that appropriate contract and incentive mechanisms may be achieved in CLSC. Govindan and Popiuc [28], De Giovanni [29], and Xiao, Yang, and Shen [30] investigate a revenue-sharing contract to coordinate a CLSC and show that it always allows better economical and environmental performance. However, Cachon and Lariviere [31] show us that a revenue-sharing contract has drawbacks when applyied in industries when chain competition occurs. De Giovanni and Roselli [32] show that an advertising-support strategy may overcome the drawbacks of the revenue-sharing contracts. Based on this, our research investigates a dynamic dual-channel closed-loop supply chain (CLSC), where a manufacturer as a leader directs both traditional retailer channels and e-tail (online) channels and collects used/end-of-life products from the retailer. In addition, both the manufacturer and the retailer invest in green advertising to educate customers and influence their awareness about environmental issues and ultimately promote customers' return rate of used/end-of-life products.

There are two streams of thought about advertising incentive mechanisms, static game frameworks, and dynamic game frameworks. Hong et al., [33] built a static Stackelberg game model to investigate the optimal decisions of local advertising, used-product collection, and pricing in centralized and decentralized CLSC. Xie et al., [34] studied the static centralized and decentralized dual-channel CLSC based on the advertising incentive and recycling rate of products. Because CLSC is a dynamic phenomenon, Savaskan et al., [20] stated that a CLSC should be investigated dynamically, because the influence of a dynamic return rate changes the players' strategies and the channel's outcomes. De Giovanni [24] analyzed a single manufacturer and a single retailer in a dynamic closed-loop supply chain (CLSC), where both of the players invest in green advertising. He shows that the green-advertising investment has a positive impact on the CLSC by implementing a reverse revenue-sharing contract. De Giovanni et al., [25] investigated a dynamic CLSC where a manufacturer directs a retailer's Stackelberg Equilibrium. They show that greenadvertising incentive strategies may stimulate higher economic, social, and environmental outcomes. De Giovanni et al., [26] shows a joint maximization green-advertising incentive is always more effective for the success of a dynamic CLSC. However, all of the literature mentioned above is focused on one manufacturer and one retailer. Little of the literature has focused on competitive models [26], and even fewer on the manufacturer directing a retailer channel and online channel as well. Therefore, we built three different dynamic green-advertising incentive strategies: a non-co-op green-advertising model (NGA-Model), where both of the players invest in green advertising separately; a co-op green-advertising model (CGA-Model), where the manufacturer supports part of the retailer's greenadvertising cost and incentivizes it to increase its green-advertising investments; and a bilateral co-op green-advertising model (BGA-Model), where the manufacturer and retailer support part of each other's green-advertising costs to incentivize each other to increase their green-advertising investments. Our purpose is to investigate which green-advertising coordination incentive mechanism is the most effective for the success of a CLSC. We make three main contributions, as follows.

1. Our paper models three different dynamic green-advertising incentive mechanisms. Previous green-advertising research in CLSC has focused on static $[20,35]$ or on dynamic 
models with one manufacturer and one retailer. Little of the literature has focused on competitive models [26] and even fewer on the manufacturer directing a retailer channel and online channel as well. Therefore, research in dynamic cooperative green-advertising incentives is still missing.

2. In all of the literature mentioned above, either one player designs an incentive mechanism for both players, or both the players design incentive mechanisms separately. In our case, we design a support-incentive mechanism for the players to stimulate each other to increase their green-advertising investments.

3. This is the first paper to compare three different cooperative green-advertising incentive strategies in CLSC.

Our findings show that the implementation of cooperative green-advertising incentive strategies always causes consumers to return more used/end-of-life products and to save raw materials, increases consumers' willingness to purchase, and ultimately achieves the goals of profit maximization and sustainable development. We especially ask the following questions:

1. What is the optimal pricing and green-advertising incentive decision policy of the dual CLSC?

2. How do the green-advertising incentive mechanisms affect the customers' environmental awareness and product-return rate?

3. Among these three strategies, which one is the best for achieving the challenge of environmental, economic and social performance?

The reminder of this paper is organized as follows. Section 2 develops the greenadvertising incentive models. Section 3 characterizes three green-advertising feedback equilibrium scenarios in a CLSC system. The numerical analysis of green-advertising incentive strategies is reported in Section 4. Conclusions and suggests for future research are summarized in Section 5.

\section{Model}

Let's consider a green-advertising coordination incentive mechanism of CLSC that can be illustrated as in Figure 1, where the manufacturer (player $M$ ) sells products to consumers through a newly added online channel at an online price, $p_{1}$. Meanwhile, the manufacturer also sells products to the retailer at a wholesale price, $\omega(t)$, and the retailer decides price $p_{2}$ for the consumers. Denote $R(t)$ to be the return rate of used/end-of-life products in a reverse logistic channel from consumers to retailer. In addition, player $M$ decides the online green-advertising efforts, $\mu_{1}$ as well as the participation rate, $\eta_{1}\left(0 \leq \eta_{1} \leq 1\right)$, as an incentive of the retailer's green-advertising expenditure. The retailer decides the traditional green-advertising efforts, $\mu_{2}$, as well as the participation rate, $\eta_{2}\left(0 \leq \eta_{2} \leq 1\right)$, as an incentive of the manufacturer's green-advertising expenditure. Green advertising could increase peoples' awareness about environmental issues that could encourage more customers to return their used/end-of-life products. We assume there is no difference between a manufactured and a remanufactured product. 


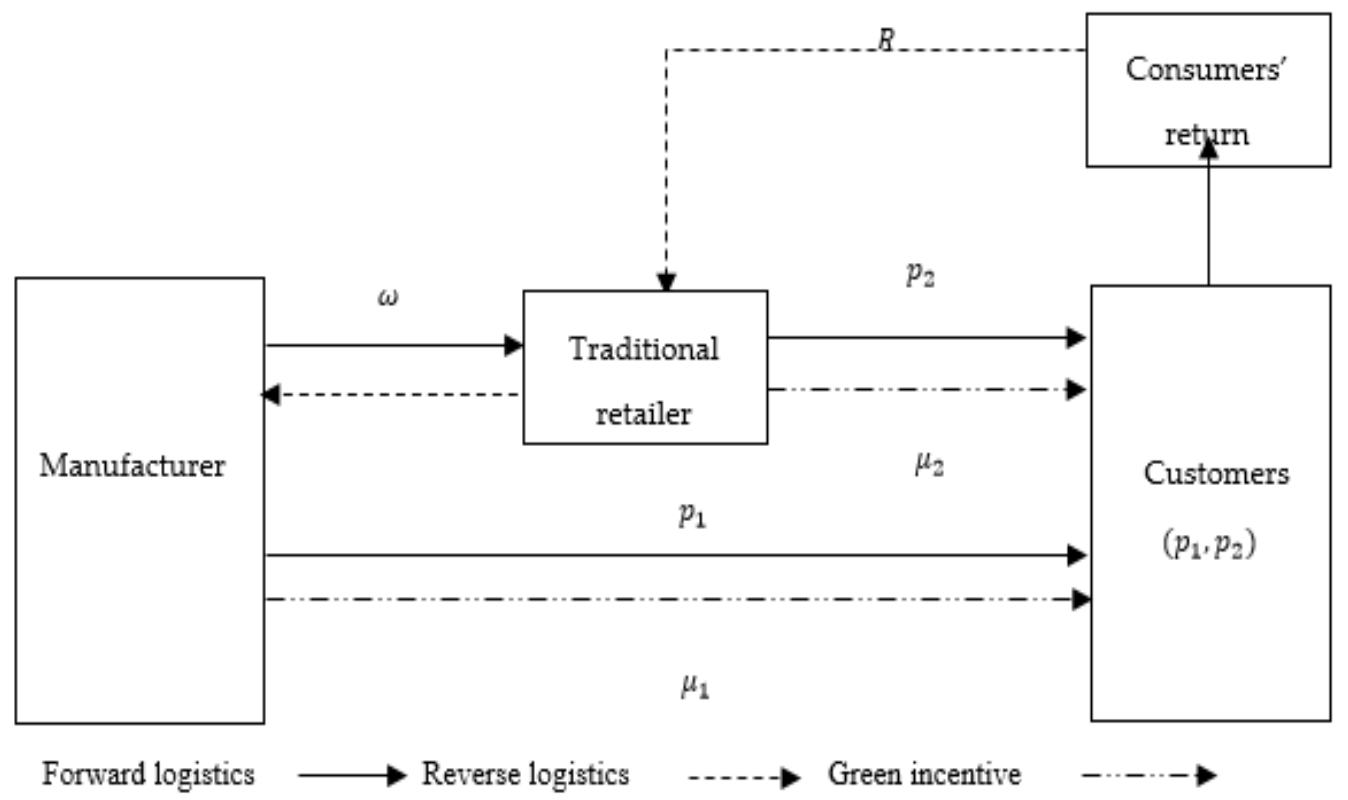

Figure 1. The dual-channel CLSC Distribution

In order to capture the dynamic return rate of the CLSL system, we use the advertising model of Giovanni et al., [25], which is the extended and evolved version of Nerlove and Arrow [36]. The green-advertising strategies contribute to the build-up of the dynamic return rate, which can be modeled as follows:

$$
\dot{R}(t)=\rho_{1} \mu_{1}(t)+\rho_{2} \mu_{2}(t)-\delta R(t), \quad R(0)=R_{0} \geq 0 .
$$

where $\rho_{1}>0$ is the effect of the manufacturer's green advertising on returns, $\rho_{2}>0$ is the effect of the retailer's green advertising on returns, $\delta>0$ is the decay rate of player $M$ loses returns. We assume that demands depend on the prices and return rate; therefore, the demands at any instant of time can be extended as follows:

$$
\begin{aligned}
& D_{1}\left(p_{1}(t), p_{2}(t), R(t)\right)=a \theta \sqrt{R(t)}-b_{1} p_{1}(t)+\beta p_{2}(t) \\
& D_{2}\left(p_{1}(t), p_{2}(t), R(t)\right)=a(1-\theta) \sqrt{R(t)}-b_{2} p_{2}(t)+\beta p_{1}(t) .
\end{aligned}
$$

where $D_{1}$ and $D_{2}$ denote the market demands of the online and offline channels, respectively; $b_{1}, b_{2}$ are nonnegative constants representing the coefficients of self-price elasticity; $a>0$ is the baseline demand; and $\beta$ is a nonnegative constant representing the degree of brand differentiation between the traditional and online channels. In addition, both of the channel sell an identical product; so the degree of brand differentiation between the channels, $\beta=1$. As in Yan et al. [37], we assume that the self-price has a greater effect than others, and we assume $b_{1}=b_{2}=b>\beta$; so that $D(t)=D_{1}(t)+D_{2}(t)=a \sqrt{R(t)}-$ $(b-1)\left(p_{1}(t)+p_{2}(t)\right)$.

Let $\lambda=b-1,0 \leq \theta \leq 1$ represent the percentage of accumulated dynamic returns going to the channel members. The green-advertising costs are assumed to have convex and increasing functions as:

$$
C\left(\mu_{i}(t)\right)=\frac{1}{2} \mu_{i}^{2}(t), \quad i=1,2
$$


The manufacturer's objective function in an infinite-time horizon CLSC system can be extended as:

$$
\begin{gathered}
J_{M}=\operatorname{Max} \int_{0}^{\infty} e^{-r t}\left\{\left(p_{1}(t)-c\right) D_{1}(t)+(\omega(t)-c) D_{2}(t)+D(t)\left(\Delta_{1}-c\right) \gamma \sqrt{R(t)}\right. \\
\left.-\frac{\left(1-\eta_{2}(t)\right)}{2} \mu_{1}^{2}(t)-\frac{\eta_{1}(t)}{2} \mu_{2}^{2}(t)\right\} d t
\end{gathered}
$$

And the retailer's objective function is:

$$
\begin{aligned}
J_{R}=\operatorname{Max} \int_{0}^{\infty} & e^{-r t}\left\{\left(p_{2}(t)-\omega(t)\right) D_{2}(t)+D(t)\left(\Delta_{2}-c\right) \gamma \sqrt{R(t)}-\frac{\left(1-\eta_{1}(t)\right)}{2} \mu_{2}^{2}(t)\right. \\
& \left.-\frac{\eta_{2}(t)}{2} \mu_{1}^{2}(t)\right\} d t .
\end{aligned}
$$

where $\gamma>0$ is an adjusting parameter, $r>0$ is the discount rate, $\Delta_{1}$ represents the cost saving from the remanufacturer, and $\Delta_{2}$ represents the retailer's profits from recycling the used/end-of-life products.

\section{Equilibria}

\subsection{NGA Feedback Equilibria}

In this section, we assume that both of the channel players decide their green-advertising activities independently and that there is no coordination with each other. which means $\eta_{1}(t)=\eta_{1}(t)=0$. Without loss of generality, we omit the time argument and assume that $c=0$. Proposition 1 demonstrates our main results for the NGA Model.

Proposition 1. Under the NGA Model, feedback equilibrium price strategies and greenadvertising decisions are given by:

$$
\begin{gathered}
p_{1}^{N G A}=\frac{N_{2} \sqrt{R^{N G A}}}{2\left(b^{2}-1\right)}, \\
\omega^{N G A}=\frac{N_{3} \sqrt{R^{N G A}}}{2 b\left(b^{2}-1\right)}, \\
p_{2}^{N G A}=\frac{N_{4} \sqrt{R^{N G A}}}{4 b\left(b^{2}-1\right)}, \\
\mu_{1}^{N G A}=f_{1} \rho_{1}, \\
\mu_{2}^{N G A}=f_{3} \rho_{2},
\end{gathered}
$$

where the parameters $f_{1}, f_{2}, f_{3}, f_{4}$ are the coefficients of the value function.

$$
\begin{aligned}
& V_{m}^{N G A}=f_{1} R^{N G A}+f_{2}, \\
& V_{r}^{N G A}=f_{3} R^{N G A}+f_{4} .
\end{aligned}
$$

Proofs are given in Appendix 1. 
By inserting $\mu_{1}^{N G A}$ and $\mu_{2}^{N G A}$ into equation (1), we can get the dynamic return rate under a non-co-op green-advertising incentive strategy CLSC system as follows:

$$
R_{\infty}^{N G A}=\rho_{1}^{2} f_{1}+\rho_{2}^{2} f_{3} .
$$

\subsection{CGA Feedback Equilibria}

In this scenario, we consider a unilateral co-op green-advertising incentive strategy in the CLSC system, which means that, in this part, the manufacturer reimburses part of the retailer's green-advertising cost $\left(\eta_{1} \neq 0\right)$, which could stimulate and influence the retailer's green-advertising decisions, ultimately promoting the whole channel's return rate and profits. Proposition 2 characterizes our main results under the CGA Model.

Proposition 2. Under the CGA Model, the feedback equilibrium price strategies and greenadvertising decisions are given by:

$$
\begin{gathered}
p_{1}^{C G A}=\frac{N_{2} \sqrt{R^{C G A}}}{2\left(b^{2}-1\right)}, \\
\omega^{C G A}=\frac{N_{3} \sqrt{R^{C G A}}}{2 b\left(b^{2}-1\right)}, \\
p_{2}^{C G A}=\frac{N_{4} \sqrt{R^{C G A}}}{4 b\left(b^{2}-1\right)}, \\
\mu_{1}^{C G A}=g_{1} \rho_{1}, \\
\mu_{2}^{C G A}=\frac{\rho_{2}\left(2 g_{1}+g_{3}\right)}{2}, \\
\eta_{1}^{C G A}=\frac{2 V_{m}^{N G A \prime}-V_{r}^{N G A \prime}}{2 V_{m}^{N G A \prime}+V_{r}^{N G A \prime}}=\frac{2 g_{1}-g_{3}}{2 g_{1}+g_{3}} .
\end{gathered}
$$

where the parameters $g_{1}, g_{2}, g_{3}, g_{4}$ are the coefficients of the value function.

$$
\begin{aligned}
& V_{m}^{C G A}=g_{1} R^{C G A}+g_{2}, \\
& V_{r}^{C G A}=g_{3} R^{C G A}+g_{4} .
\end{aligned}
$$

Proofs are given in Appendix 2.

As in the previous scenario, the optimal price strategies are goodwill-state-dependent. In contrast, the advertising decisions are constant. The above results also show us that, when the manufacturer's margin is more than half of the retailer's, he will support the retailer's green-advertising strategy, otherwise, he will support nothing. On the other hand, he will support all of the green-advertising cost if the retailer's margin is zero. By inserting $\mu_{1}^{C G A}$ and $\mu_{2}^{C G A}$ into equation (1), we can get the accumulation of consumer dynamic return rate under a unilateral co-op green-advertising incentive strategy CLSC system as follows:

$$
R_{\infty}^{C G A}=\frac{2 \rho_{1}^{2} g_{1}+\rho_{2}^{2}\left(2 g_{1}+g_{3}\right)}{2 \delta} .
$$




\subsection{BGA Feedback Equilibria}

In this scenario, consistent with Zhang et al., [[37]], we consider a bilateral greenadvertising promotion strategy in the CLSC system, which means that, in this part, the manufacturer and retailer reimburse part of each other's green-advertising cost $\left(\eta_{1} \neq\right.$ $0, \eta_{2} \neq 0$,) to stimulate and influence the retailer's green-advertising decisions, which ultimately promotes the whole channel's return rate and profits. Proposition 3 characterizes our main results for the bilateral green-advertising promotion strategy.

Proposition 3. Under the $B G A$ Model, the feedback equilibrium price strategies and greenadvertising decisions are given by:

$$
\begin{gathered}
p_{1}^{B G A}=\frac{N_{2} \sqrt{R^{B G A}}}{2\left(b^{2}-1\right)}, \\
\omega^{B G A}=\frac{N_{3} \sqrt{R^{B G A}}}{2 b\left(b^{2}-1\right)}, \\
p_{2}^{B G A}=\frac{N_{4} \sqrt{R^{B G A}}}{4 b\left(b^{2}-1\right)}, \\
\mu_{1}^{B G A}=\frac{h_{3} \rho_{1}}{\eta_{2}^{B G A}}, \\
\eta_{1}^{B G A}=\frac{h_{3} \rho_{2}}{1-\eta_{1}^{B G A}}, \\
\eta_{2}^{B G A}=\frac{2 V_{m}^{B G A \prime}-V_{r}^{B G A \prime}}{2 V_{m}^{B G A^{\prime}}+V_{r}^{B G A^{\prime}}}=\frac{2 h_{1}-h_{3}}{2 h_{1}+h_{3}} . \\
2 V_{m}^{B G V^{\prime}}+V_{r}^{B G A^{\prime}}=\frac{2 h_{3}}{2 h_{1}+h_{3}} .
\end{gathered}
$$

where the parameters $h_{1}, h_{2}, h_{3}, h_{4}$ are the coefficients of the value function.

$$
\begin{gathered}
V_{m}^{B G A}=h_{1} R^{B G A}+h_{2}, \\
V_{r}^{B G A}=h_{3} R^{B G A}+h_{4} .
\end{gathered}
$$

As in the previous scenarios, the optimal price strategies depend on the dynamic return rate. In contrast, the green-advertising decisions are constant. The above results also show us that, when the manufacturer's margin is more than half of the retailer's, he will support the retailer's green-advertising strategy; otherwise, he will support nothing. On the other hand, he will support all of the green-advertising cost if the retailer margin is zero. Meanwhile, the retailer will always support the manufacturer's green-advertising strategy if the retailer's margin is not zero.

By inserting (27)-(30) into equation (1), we can get the accumulation of consumer dynamic return rate under a bilateral co-op green-advertising incentive strategy CLSC system as follows: 


$$
R_{\infty}^{B G A}=\frac{\left(2 h_{1}+h_{3}\right)\left(\rho_{1}^{2}+\rho_{2}^{2}\right)}{2 \delta} .
$$

\section{Numerical Analysis}

In this section, we consider numerical examples to investigate the three different greenadvertising CLSC models' optimal operations and decisions. Our purpose is to check, through the numerically presented theoretical results and the managerial insights provided by sensitivity analysis, which green-advertising incentive strategy is the best for improving channel profits and reducing the conflict.

In order to get the numerical results, all of the parameters are assumed to be exogenous, according to previous research $[10,12,39,40]$, the parameters are considered as follows: $\rho_{1}=0.5 ; \rho_{2}=0.5 ; \delta=0.1 ; \lambda=0.03 ; \Delta_{1}=0.3 ; \Delta_{2}=0.1 ; r=0.1 ; b=1.2 ;$

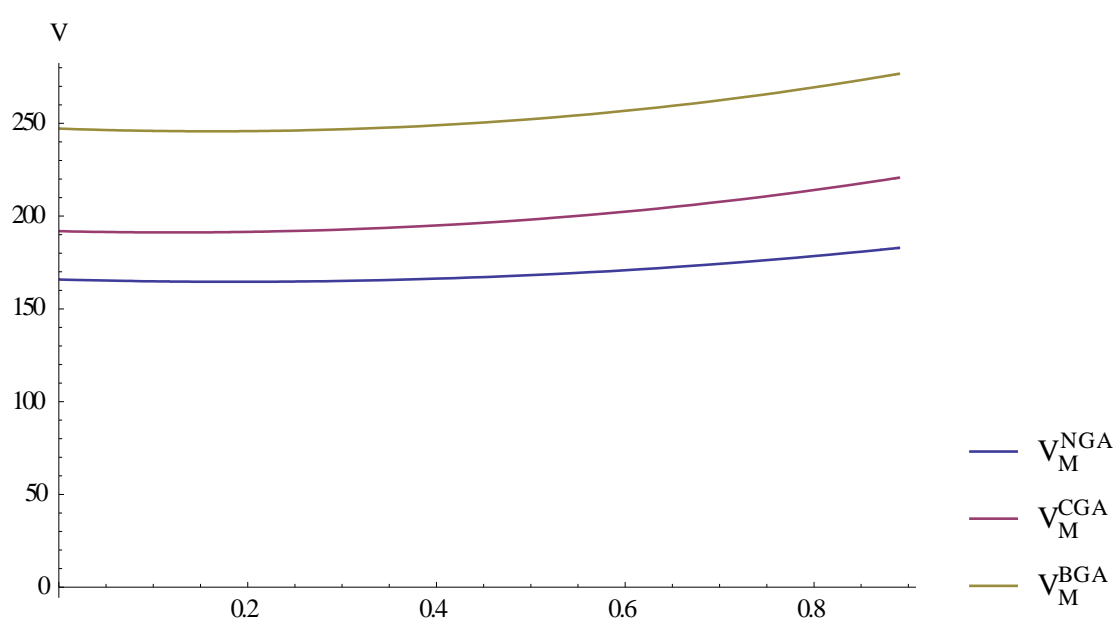

(a) $\theta$ vs. $V_{M}$

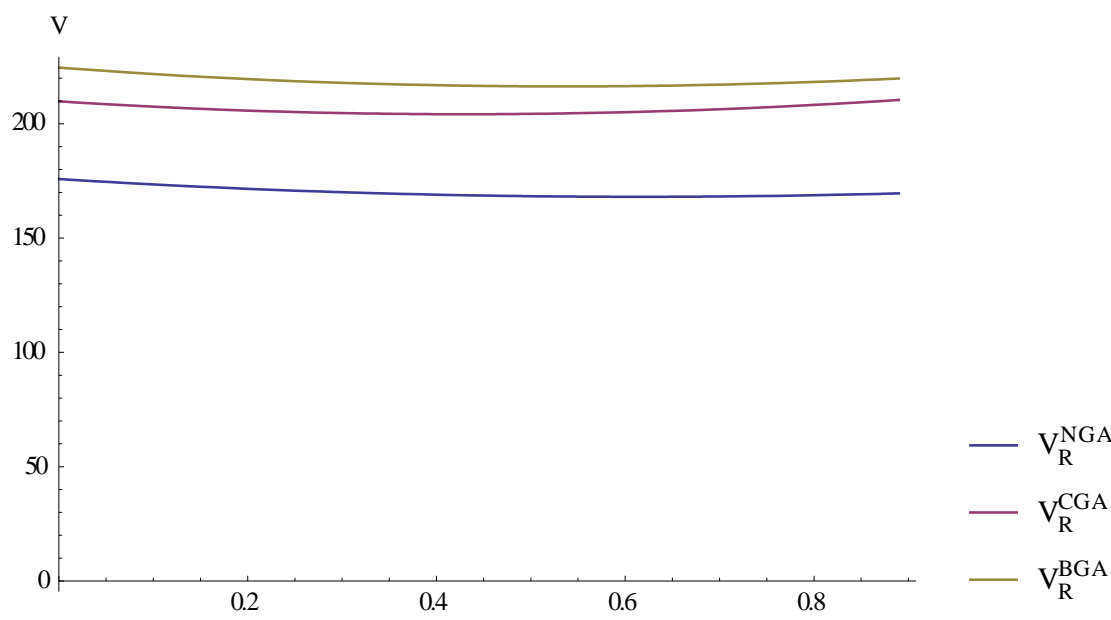

(b) $\theta$ vs. $V_{R}$

Figure 2. Relationship between the Players' Profits and MarketSharing Parameter $\boldsymbol{\theta}$

As the market-sharing parameter $\theta$ increases, that is, as the customers prefer shopping online more, the retailer's profits decrease as his market sharing decrease (Figure 2b), the manufacturer's profit increase due to his demand increase (Figure 2a). 


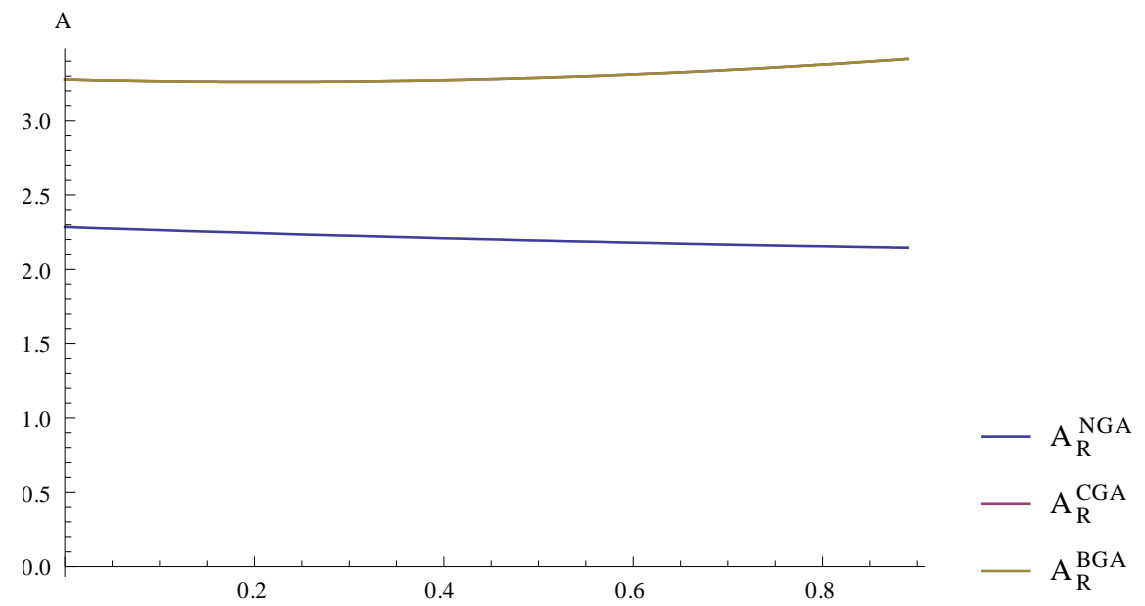

Figure 3. Relationship between Retailer's Green-Advertising Incentive and Market-Sharing Parameter $\boldsymbol{\theta}$

Figure 3 depicts that, as the market-sharing parameter $\theta$ increases, the retailer would reduce his green-advertising investment under the non-co-op green-advertising incentive strategy. However, under a co-op green-advertising incentive strategy, because the manufacturer's profits increase, he would like to give more support to the retailer; with the subsidy from the manufacturer, the retailer could recoup his losses and would like to invest in more green advertising, which ultimately stimulates the customers' return rate and his profits. Therefore, the co-op green-advertising incentive strategy has a positive effect on the retailer's investment in green advertising. On the other hand, both the unilateral co-op green-advertising incentive strategy and the bilateral co-op green-advertising incentive strategy have the same effect on the retailer's green-advertising investment.

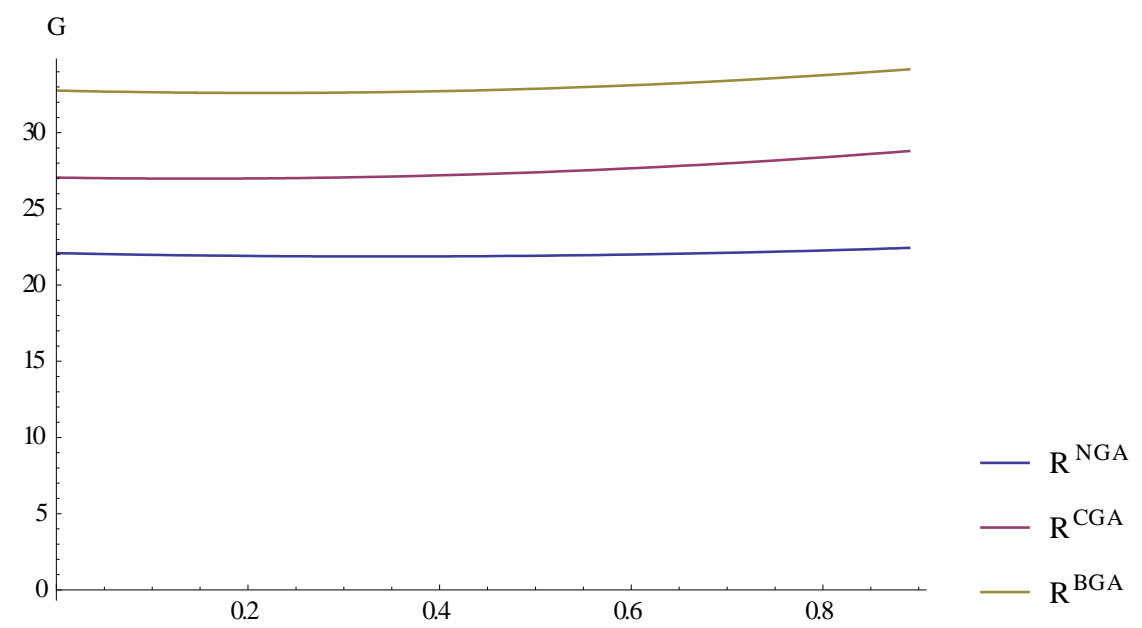

Figure 4. Relationship between Return Rate and Market-Sharing Parameter $\theta$

Figure 4 shows that, among three green-advertising incentive strategies, as the marketsharing parameter $\theta$ increases, the CLSC system has the highest return rate under a bilateral co-op green-advertising incentive strategy.

\section{Conclusions}

In this research, we have investigated three different green-advertising incentive strategies models in a dynamic dual-channel closed-loop supply chain (CLSC): a non-co- 
op green-advertising incentive model, a unilateral co-op green-advertising incentive model, and a bilateral co-op green-advertising incentive model. In our research, we compute the optimal price and advertising strategies, payoffs, and dynamic return rate of consumers. Our main results can be summarized as follows:

(i). Among these three green-advertising incentive strategies scenarios, the optimal price strategies are state-dependent. In contrast, the green-advertising decisions are stateindependent;

(ii). In the newly added online multichannel situation, green-advertising incentive strategies have the positive effect of achieving the goals of profit maximization and sustainable development, and ultimately leading to a Pareto improving;

(iii). The outcomes for the manufacturer and retailer are always better under the bilateral co-op green-advertising incentive model.

Future research can be extended in several directions, such as service decisions and information sharing. Second, it should be much more interesting to use different types of contracts, such as revenue sharing and paybacks in our model. Third, we assume there is no difference between manufactured and remanufactured products; however, the remanufactured product could not be as good as the new one, which should be considered in future research.

Supplementary Materials: The [Mathematica Untitled.nb] code used to support the findings of this study have been deposited in the [Scientific Data recommended repositories] repository [doi].

\section{Appendix 1}

We first let $V_{m}^{N G A}(G), V_{r}^{N G A}(G)$ be value functions of a manufacturer directing both traditional retailer channels and online channels based on dynamic return rate in a continuously differentiable function. The channel members' HJB equations for value functions under the NGA Model can be written as:

$$
\begin{aligned}
& r V_{m}^{N G A}=\left\{p_{1}^{N G A}\left(\theta \sqrt{R^{N G A}}-b p_{1}^{N G A}+p_{2}^{N G A}\right)\right. \\
& +\omega^{N G A}\left[(1-\theta) \sqrt{R^{N G A}}-b p_{2}^{N G A}+p_{1}^{N G A}\right)+\Delta_{1} \gamma \sqrt{R^{N G A}}\left(\sqrt{R^{N G A}}\right. \\
& \left.-\lambda p_{1}^{N G A}-\lambda p_{2}^{N G A}\right)-\frac{1}{2}\left(\mu_{1}^{N G A}\right)^{2} \\
& \left.+V_{m}^{N G A^{\prime}}\left(\rho_{1} \mu_{1}^{N G A}+\rho_{2} \mu_{2}^{N G A}-\delta R^{N G A}\right)\right\} \\
r V_{r}^{N G A}=\{ & \left(p_{2}^{N G A}-\omega^{N G A}\right)\left[(1-\theta) \sqrt{R^{N G A}}-b p_{2}^{N G A}+p_{1}^{N G A}\right]+\Delta_{2} \gamma \sqrt{R^{N G A}}\left(\sqrt{R^{N G A}}\right. \\
& \left.-\lambda p_{1}^{N G A}-\lambda p_{2}^{N G A}\right)-\frac{1}{2}\left(\mu_{2}^{N G A}\right)^{2} \\
& \left.+V_{r}^{N G A^{\prime}}\left(\rho_{1} \mu_{1}^{N G A}+\rho_{2} \mu_{2}^{N G A}-\delta R^{N G A}\right)\right\}
\end{aligned}
$$

Take the first-order conditions with respect to $p_{2}^{N G A}$ and $\mu_{2}^{N G A}$ to maximization the retailer as:

$$
p_{2}^{N G A}=\frac{\left[(1-\theta)-\lambda \Delta_{2}\right] \sqrt{R^{N G A}}+b p_{1}^{N G A}+b \omega^{N G A}}{2 b}
$$




$$
\mu_{2}^{N G A}=V_{r}^{N G A^{\prime}} \rho_{2}
$$

Substituting (36) into (34), we obtain:

$$
\begin{aligned}
r V_{m}^{N G A}=\left\{p_{1}^{N G A}\right. & \left(\theta \sqrt{R^{N G A}}-b p_{1}^{N G A}+\frac{\left[(1-\theta)-\lambda \Delta_{2}\right] \sqrt{R^{N G A}}+b p_{1}^{N G A}+b \omega^{N G A}}{2 b}\right) \\
& +\omega^{N G A}\left[(1-\theta) \sqrt{R^{N G A}}-b \frac{\left[(1-\theta)-\lambda \Delta_{2}\right] \sqrt{R^{N G A}}+b p_{1}^{N G A}+b \omega^{N G A}}{2 b}\right. \\
& \left.+p_{1}^{N G A}\right)+\Delta_{1} \gamma \sqrt{R^{N G A}}\left(\sqrt{R^{N G A}}-\lambda p_{1}^{N G A}\right. \\
& -\lambda \frac{\left.\left[(1-\theta)-\lambda \Delta_{2}\right] \sqrt{R^{N G A}}+b p_{1}^{N G A}+b \omega^{N G A}\right)-\frac{1}{2}\left(\mu_{1}^{N G A}\right)^{2}}{2 b} \\
& \left.+V_{m}^{N G A^{\prime}}\left(\rho_{1} \mu_{1}^{N G A}+\rho_{2} \mu_{2}^{N G A}-\delta R^{N G A}\right)\right\}
\end{aligned}
$$

Taking the first-order conditions with respect to $p_{1}^{N G A}, \omega^{N G A}$ and $\mu_{1}^{N G A}$ to maximize the manufacturer, we obtain

$$
\begin{aligned}
& p_{1}^{N G A}=\frac{N_{2} \sqrt{R^{N G A}}}{2\left(b^{2}-1\right)}, \\
& \omega^{N G A}=\frac{N_{3} \sqrt{R^{N G A}}}{2 b\left(b^{2}-1\right)}, \\
& \mu_{1}^{N G A}=\rho_{1} V_{r}^{N G A^{\prime}} .
\end{aligned}
$$

Then, substituting (39) and (40) into (36), we obtain

$$
p_{2}^{N G A}=\frac{N_{4} \sqrt{R^{N G A}}}{4 b\left(b^{2}-1\right)}
$$

Here,

$$
\begin{gathered}
N_{1}=(1-\theta)-\lambda \Delta_{2}, \\
N_{2}=(1-\theta)+b \theta-\lambda \Delta_{1}(1+b), \\
N_{3}=(b-1)(b+1)(1-\theta)-b \theta(b-1)+b^{2}\left(1-N_{1}\right)-\lambda \Delta_{1}(1+b)+N_{1}, \\
N_{4}=2\left(b^{2}-1\right) N_{1}+N_{3}+N_{2} .
\end{gathered}
$$

We look for the linear value functions,

$$
\begin{aligned}
& V_{m}^{N G A}=f_{1} R^{N G A}+f_{2}, V_{m}^{N G A^{\prime}}=f_{1}, \\
& V_{r}^{N G A}=f_{3} R^{N G A}+f_{4}, V_{r}^{N G A^{\prime}}=f_{3} .
\end{aligned}
$$

where $f_{1}, f_{2}, f_{3}, f_{4}$ are the parameters. Substituting (37) and (39)-(42) into HJB equations and simultaneous equations (47) and (48), we can obtain: 


$$
\begin{aligned}
& \begin{array}{l}
4 b N_{2}\left(b^{2}-1\right) \theta-2 b^{2} N_{2}^{2}+N_{2} N_{4}+4\left(b^{2}-1\right)(1-\theta) N_{3}-N_{3} N_{4}+2 N_{2} N_{3} \\
+8 b\left(b^{2}-1\right)^{2} \Delta_{1}-4 b \lambda \Delta_{1} N_{2}\left(b^{2}-1\right)-2 \Delta_{1} \lambda N_{4}\left(b^{2}-1\right) \\
=8 b\left(b^{2}-1\right)^{2}(r+\delta) f_{1}
\end{array} \\
& \quad f_{1}^{2} \rho_{1}^{2}+2 f_{1} f_{3} \rho_{2}^{2}=2 r f_{2} \\
& 4\left(b^{2}-1\right)\left(N_{4}-2 N_{3}\right)(1-\theta)-N_{4}\left(N_{4}-2 N_{3}\right)+2 N_{2}\left(N_{4}-2 N_{3}\right)+16 b\left(b^{2}-1\right)^{2} \Delta_{2} \\
& -8 b \Delta_{2} N_{2} \lambda\left(b^{2}-1\right)-4 \Delta_{2} N_{4} \lambda\left(b^{2}-1\right. \\
& =16 b\left(b^{2}-1\right)^{2}(r+\delta) f_{3}
\end{aligned}
$$

simultaneous equations (49)-(52), we can easily obtain the parameters $f_{1}, f_{2}, f_{3}, f_{4}$. And inserting $f_{1}$ and $f_{3}$ into (37) and (41), respectively, we can easily get

$=\frac{\rho_{1}\left(\begin{array}{c}4 b^{2} N_{3}-2 b^{2} N_{2}^{2}-4 N_{3}+2 N_{2} N_{3}+N_{2} N_{4}-N_{3} N_{4} \\ -4 b N_{2} \theta+4 b^{3} N_{2} \theta+4 N_{3} \theta-4 b^{2} N_{3} \theta+8 b \triangle_{1}-16 b^{3} \triangle_{1} \\ +8 b^{5} \triangle_{1}+4 b \lambda \triangle_{1}-4 b^{3} \lambda \triangle_{1}+2 N_{4} \lambda \triangle_{1}-2 b^{2} N_{4} \lambda \triangle_{1}\end{array}\right)}{8 b\left(b^{2}-1\right)^{2}(r+\delta)}$, $\mu_{1}^{N G A}$

$=\frac{\rho_{2}\left(\begin{array}{c}8 N_{3}-8 b^{2} N_{3}-2 N_{2} N_{3}-4 N_{4}+4 b^{2} N_{4}+2 N_{2} N_{4}+2 N_{3} N_{4}-N_{4}^{2} \\ -8 N_{3} \theta+8 b^{2} N_{3} \theta+4 N_{4} \theta-4 b^{2} N_{4} \theta+16 b \triangle_{2}-32 b^{3} \triangle_{2} \\ +16 b^{5} \triangle_{2}+8 b N_{2} \lambda \triangle_{2}-8 b^{3} N_{2} \lambda \triangle_{2}+4 N_{4} \lambda \triangle_{2}-4 b^{2} N_{4} \lambda \triangle_{2}\end{array}\right)}{16 b\left(b^{2}-1\right)^{2}(r+\delta)}$

\section{Appendix 2}

We first let $V_{m}^{C G A}(G), V_{r}^{C G A}(G)$ be value functions of a manufacturer directing both traditional retailer channels and online channels based on a dynamic rerurn rate in a continuously differentiable function. The channel members' HJB equations for value functions under the $C G A$-Model can be written as:

$$
\begin{gathered}
r V_{m}^{C G A}=\left\{p_{1}^{C G A}\left(\theta \sqrt{R^{C G A}}-b p_{1}^{C G A}+p_{2}^{C G A}\right)+\omega^{C G A}\left[(1-\theta) \sqrt{R^{C G A}}-b p_{2}^{C G A}+p_{1}^{C G A}\right)\right. \\
+\Delta_{1} \gamma \sqrt{R^{C G A}}\left(\sqrt{R^{C G A}}-\lambda p_{1}^{C G A}-\lambda p_{2}^{C G A}\right)-\frac{1}{2}\left(\mu_{1}^{C G A}\right)^{2}-\frac{\eta_{1}^{C G A}}{2}\left(\mu_{2}^{C G A}\right)^{2} \\
\left.+V_{m}^{C G A^{\prime}}\left(\rho_{1} \mu_{1}^{C G A}+\rho_{2} \mu_{2}^{C G A}-\delta R^{C G A}\right)\right\}, \\
r V_{r}^{C G A}=\left\{\left(p_{2}^{C G A}-\omega^{C G A}\right)\left[(1-\theta) \sqrt{R^{C G A}}-b p_{2}^{C G A}+p_{1}^{C G A}\right]+\Delta_{2} \gamma \sqrt{R^{C G A}}\left(\sqrt{R^{C G A}}\right.\right. \\
\left.\quad-\lambda p_{1}^{C G A}-\lambda p_{2}^{C G A}\right)-\frac{\left(1-\eta_{1}^{C G A}\right)}{2}\left(\mu_{2}^{C G A}\right)^{2} \\
\left.+V_{r}^{C G A^{\prime}}\left(\rho_{1} \mu_{1}^{C G A}+\rho_{2} \mu_{2}^{C G A}-\delta R^{C G A}\right)\right\} .
\end{gathered}
$$

We take the first-order conditions with respect to $p_{2}^{C G A}$ and $\mu_{2}^{C G A}$ to maximize the retailer as: 


$$
\begin{gathered}
p_{2}^{C G A}=\frac{\left[(1-\theta)-\lambda \Delta_{2}\right] \sqrt{R^{C G A}}+b p_{1}^{C G A}+b \omega^{C G A}}{2 b}, \\
\mu_{2}^{C G A}=\frac{V_{r}^{C G A^{\prime}} \rho_{2}}{1-\eta_{1}^{C G A}} .
\end{gathered}
$$

Substituting (57)-(58) into (55), we obtain:

$$
\begin{aligned}
r V_{m}^{C G A}=\{ & p_{1}^{C G A}\left(\theta \sqrt{R^{C G A}}-b p_{1}^{C G A}+\frac{\left[(1-\theta)-\lambda \Delta_{2}\right] \sqrt{R^{C G A}}+b p_{1}^{C G A}+b \omega^{C G A}}{2 b}\right) \\
& +\omega^{C G A}\left[(1-\theta) \sqrt{R^{C G A}}-b \frac{\left[(1-\theta)-\lambda \Delta_{2}\right] \sqrt{R^{C G A}}+b p_{1}^{C G A}+b \omega^{C G A}}{2 b}\right. \\
& \left.+p_{1}^{C G A}\right) \\
& +\Delta_{1} \gamma \sqrt{R^{C G A}}\left(\sqrt{R^{C G A}}-\lambda p_{1}^{C G A}\right. \\
& \left.-\lambda \frac{\left[(1-\theta)-\lambda \Delta_{2}\right] \sqrt{R^{C G A}}+b p_{1}^{C G A}+b \omega^{C G A}}{2 b}\right)-\frac{1}{2}\left(\mu_{1}^{C G A}\right)^{2} \\
& -\frac{\eta_{1}^{C G A}}{2}\left(\frac{V_{r}^{C G A^{\prime}} \rho_{2}}{1-\eta_{1}^{C G A}}\right)^{2} \\
& \left.+V_{m}^{C G A^{\prime}}\left(\rho_{1} \mu_{1}^{C G A}+\frac{V_{r}^{C G A^{\prime}} \rho_{2}^{2}}{1-\eta_{1}^{C G A}}-\delta R^{C G A}\right)\right\}
\end{aligned}
$$

Taking the first-order conditions with respect to $p_{1}^{C G A}, \omega^{C G A}, \mu_{1}^{C G A}$ and $\eta_{1}^{C G A}$ to maximize the manufacturer we obtain

$$
\begin{gathered}
p_{1}^{C G A}=\frac{N_{2} \sqrt{R^{C G A}}}{2\left(b^{2}-1\right)}, \\
\omega^{C G A}=\frac{N_{3} \sqrt{R^{C G A}}}{2 b\left(b^{2}-1\right)}, \\
\mu_{1}^{C G A}=\rho_{1} V_{m}^{C G A^{\prime}} . \\
\eta_{1}^{C G A}=\frac{2 V_{m}^{C G A^{\prime}}-V_{r}^{C G A^{\prime}}}{2 V_{m}^{C G A^{\prime}}+V_{r}^{C G A^{\prime}}} .
\end{gathered}
$$

Then, substituting (60) and (61) into (57), we obtain

$$
p_{2}^{C G A}=\frac{N_{4} \sqrt{R^{C G A}}}{4 b\left(b^{2}-1\right)}
$$

We look for the linear value functions,

$$
\begin{aligned}
& V_{m}^{C G A}=g_{1} R^{C G A}+g_{2}, V_{m}^{C G A^{\prime}}=g_{1}, \\
& V_{r}^{C G A}=g_{3} R^{C G A}+g_{4}, V_{r}^{C G A^{\prime}}=g_{3} .
\end{aligned}
$$


where $g_{1}, g_{2}, g_{3}, g_{4}$ are the parameters. Substituting (58) and (60)-(64) into HJB equations and simultaneous equations (65) and (66), we can obtain:

$$
\begin{gathered}
4 b N_{2}\left(b^{2}-1\right) \theta-2 b^{2} N_{2}^{2}+N_{2} N_{4}+4\left(b^{2}-1\right)(1-\theta) N_{3}-N_{3} N_{4}+2 N_{2} N_{3} \\
\quad+8 b\left(b^{2}-1\right)^{2} \Delta_{1}-4 b \lambda \Delta_{1} N_{2}\left(b^{2}-1\right)-2 \Delta_{1} \lambda N_{4}\left(b^{2}-1\right) \\
=8 b\left(b^{2}-1\right)^{2}(r+\delta) g_{1}, \\
4 g_{1}^{2} \rho_{1}^{2}-\rho_{2}^{2}\left(2 g_{1}+g_{3}\right)\left(2 g_{1}-g_{3}\right)+4 g_{1} \rho_{2}^{2}\left(2 g_{1}+g_{3}\right)=8 r g_{2} \\
4\left(b^{2}-1\right)\left(N_{4}-2 N_{3}\right)(1-\theta)-N_{4}\left(N_{4}-2 N_{3}\right)+2 N_{2}\left(N_{4}-2 N_{3}\right)+16 b\left(b^{2}-1\right)^{2} \Delta_{2} \\
-8 b \Delta_{2} N_{2} \lambda\left(b^{2}-1\right)-4 \Delta_{2} N_{4} \lambda\left(b^{2}-1\right. \\
=16 b\left(b^{2}-1\right)^{2}(r+\delta) g_{3} \\
g_{3} \rho_{2}^{2}\left(2 g_{1}+g_{3}\right)+4 g_{1} g_{3} \rho_{1}^{2}=4 r g_{4} .
\end{gathered}
$$

simultaneous equations (67)-(70), we can easily obtain the parameters $g_{1}, g_{2}, g_{3}, g_{4}$. And inserting $g_{1}$ and $g_{3}$ into (58), (62) and (63), respectively, we can easily get

$$
=\frac{\rho_{1}\left(\begin{array}{c}
4 b^{2} N_{3}-2 b^{2} N_{2}^{2}-4 N_{3}+2 N_{2} N_{3}+N_{2} N_{4}-N_{3} N_{4} \\
-4 b N_{2} \theta+4 b^{3} N_{2} \theta+4 N_{3} \theta-4 b^{2} N_{3} \theta+8 b \triangle_{1}-16 b^{3} \triangle_{1} \\
+8 b^{5} \triangle_{1}+4 b \lambda \triangle_{1}-4 b^{3} \lambda \triangle_{1}+2 N_{4} \lambda \triangle_{1}-2 b^{2} N_{4} \lambda \triangle_{1}
\end{array}\right)}{8 b\left(b^{2}-1\right)^{2}(r+\delta)},
$$

$$
\begin{aligned}
&= \frac{\mu_{2}\left(\begin{array}{c}
\text { CGA } \\
4 b^{2} N_{3}-2 b^{2} N_{2}^{2}-4 N_{3}+2 N_{2} N_{3}+N_{2} N_{4}-N_{3} N_{4} \\
-4 b N_{2} \theta+4 b^{3} N_{2} \theta+4 N_{3} \theta-4 b^{2} N_{3} \theta+8 b \triangle_{1}-16 b^{3} \triangle_{1} \\
+8 b^{5} \triangle_{1}+4 b \lambda \triangle_{1}-4 b^{3} \lambda \triangle_{1}+2 N_{4} \lambda \triangle_{1}-2 b^{2} N_{4} \lambda \triangle_{1}
\end{array}\right)}{8 b\left(b^{2}-1\right)^{2}(r+\delta)} \\
&+\frac{\rho_{2}\left(\begin{array}{c}
8 N_{3}-8 b^{2} N_{3}-2 N_{2} N_{3}-4 N_{4}+4 b^{2} N_{4}+2 N_{2} N_{4}+2 N_{3} N_{4}-N_{4}^{2} \\
-8 N_{3} \theta+8 b^{2} N_{3} \theta+4 N_{4} \theta-4 b^{2} N_{4} \theta+16 b \triangle_{2}-32 b^{3} \triangle_{2} \\
+16 b^{5} \triangle_{2}+8 b N_{2} \lambda \triangle_{2}-8 b^{3} N_{2} \lambda \triangle_{2}+4 N_{4} \lambda \triangle_{2}-4 b^{2} N_{4} \lambda \triangle_{2}
\end{array}\right)}{32 b\left(b^{2}-1\right)^{2}(r+\delta)},
\end{aligned}
$$

$$
=\frac{\left(\begin{array}{c}
-8 b^{2} N_{2}^{2}-24 N_{3}+24 b^{2} N_{3}+10 N_{2} N_{3}+4 N_{4}-4 b^{2} N_{4}+2 N_{2} N_{4}-6 N_{3} N_{4} \\
+N 4^{2}-16 b N_{2} \theta+16 b^{3} N_{2} \theta+24 N_{3} \theta-24 b^{2} N_{3} \theta-4 N_{4} \theta+4 b^{2} N_{4} \theta+32 b \triangle_{1} \\
-64 b^{3} \triangle_{1}+32 b^{5} \triangle_{1}+16 b \lambda \triangle_{1}-16 b^{3} \lambda \triangle_{1}+8 N_{4} \lambda \triangle_{1}-8 b^{2} N_{4} \lambda \triangle_{1}-16 b \triangle_{2} \\
+32 b^{3} \triangle_{2}-16 b^{5} \triangle_{2}-8 b N_{2} \lambda \triangle_{2}+8 b^{3} N_{2} \lambda \triangle_{2}-4 N_{4} \lambda \triangle_{2}+4 b^{2} N_{4} \lambda \triangle_{2}
\end{array}\right)}{\left(\begin{array}{c}
-8 b^{2} N_{2}^{2}-8 N_{3}+8 b^{2} N_{3}+6 N_{2} N_{3}-4 N_{4}+4 b^{2} N_{4}+6 N_{2} N_{4}-2 N_{3} N_{4}-N 4^{2} \\
-16 b N_{2} \theta+16 b^{3} N_{2} \theta+8 N_{3} \theta-8 b^{2} N_{3} \theta+4 N_{4} \theta-4 b^{2} N_{4} \theta+32 b \triangle_{1} \\
-64 b^{3} \triangle_{1}+32 b^{5} \triangle_{1}+16 b \lambda \triangle_{1}-16 b^{3} \lambda \triangle_{1}+8 N_{4} \lambda \triangle_{1}-8 b^{2} N_{4} \lambda \triangle_{1}+16 b \triangle_{2} \\
-32 b^{3} \triangle_{2}+16 b^{5} \triangle_{2}+8 b N_{2} \lambda \triangle_{2}-8 b^{3} N_{2} \lambda \triangle_{2}+4 N_{4} \lambda \triangle_{2}-4 b^{2} N_{4} \lambda \triangle_{2}
\end{array}\right) .}
$$

\section{Appendix 3}

We first let $V_{m}^{B G A}(G), V_{r}^{B G A}(G)$ be value functions of a manufacturer directing both traditional retailer channels and online channels based on dynamic rerurn rate in a 
continuously differentiable function. The channel members' HJB equations for value functions under the BGA Model can be written as:

$$
\begin{gathered}
r V_{m}^{B G A}=\left\{p_{1}^{B G A}\left(\theta \sqrt{R^{B G A}}-b p_{1}^{B G A}+p_{2}^{B G A}\right)+\omega^{B G A}\left[(1-\theta) \sqrt{R^{B G A}}-b p_{2}^{B G A}+p_{1}^{B G A}\right)\right. \\
+\Delta_{1} \gamma \sqrt{R^{B G A}}\left(\sqrt{R^{B G A}}-\lambda p_{1}^{B G A}-\lambda p_{2}^{B G A}\right)-\frac{\left(1-\eta_{2}^{B G A}\right)}{2}\left(\mu_{1}^{B G A}\right)^{2} \\
\left.\quad-\frac{\eta_{1}^{B G A}}{2}\left(\mu_{2}^{B G A}\right)^{2}+V_{m}^{B G A^{\prime}}\left(\rho_{1} \mu_{1}^{B G A}+\rho_{2} \mu_{2}^{B G A}-\delta R^{B G A}\right)\right\} \\
r V_{r}^{B G A}=\left\{\left(p_{2}^{B G A}-\omega^{B G A}\right)\left[(1-\theta) \sqrt{R^{B G A}}-b p_{2}^{B G A}+p_{1}^{B G A}\right]\right. \\
+\Delta_{2} \gamma \sqrt{R^{B G A}}\left(\sqrt{R^{B G A}}-\lambda p_{1}^{B G A}-\lambda p_{2}^{B G A}\right)-\frac{\eta_{2}^{B G A}}{2}\left(\mu_{1}^{B G A}\right)^{2} \\
\left.-\frac{\left(1-\eta_{1}^{B G A}\right)}{2}\left(\mu_{2}^{B G A}\right)^{2}+V_{r}^{B G A^{\prime}}\left(\rho_{1} \mu_{1}^{B G A}+\rho_{2} \mu_{2}^{B G A}-\delta R^{B G A}\right)\right\}
\end{gathered}
$$

Take the first-order conditions with respect to $p_{2}^{B G A}, \mu_{1}^{B G A}$ and $\mu_{2}^{B G A}$ to maximize the retailer as:

$$
\begin{gathered}
p_{2}^{B G A}=\frac{\left[(1-\theta)-\lambda \Delta_{2}\right] \sqrt{R^{B G A}}+b p_{1}^{B G A}+b \omega^{B G A}}{2 b}, \\
\mu_{1}^{B G A}=\frac{V_{r}^{B G A^{\prime}} \rho_{1}}{\eta_{2}^{B G A}} . \\
\mu_{2}^{B G A}=\frac{V_{r}^{B G A^{\prime}} \rho_{2}}{1-\eta_{1}^{B G A} .}
\end{gathered}
$$

Substituting (76)-(78) into (74), we obtain:

$$
\begin{aligned}
r V_{m}^{B G A}=\{ & p_{1}^{B G A}\left(\theta \sqrt{R^{B G A}}-b p_{1}^{B G A}+\frac{\left[(1-\theta)-\lambda \Delta_{2}\right] \sqrt{R^{B G A}}+b p_{1}^{B G A}+b \omega^{B G A}}{2 b}\right) \\
& +\omega^{B G A}\left[(1-\theta) \sqrt{R^{B G A}}-b \frac{\left[(1-\theta)-\lambda \Delta_{2}\right] \sqrt{R^{B G A}}+b p_{1}^{B G A}+b \omega^{B G A}}{2 b}\right. \\
& \left.+p_{1}^{B G A}\right) \\
& +\Delta_{1} \gamma \sqrt{R^{B G A}}\left(\sqrt{R^{B G A}}-\lambda p_{1}^{B G A}\right. \\
& \left.-\lambda \frac{\left[(1-\theta)-\lambda \Delta_{2}\right] \sqrt{R^{B G A}}+b p_{1}^{B G A}+b \omega^{B G A}}{2 b}\right) \\
& -\frac{\left(1-\eta_{2}^{B G A}\right)}{2}\left(\frac{V_{r}^{B G A^{\prime}} \rho_{1}}{\eta_{2}^{B G A}}\right)^{2}-\frac{\eta_{1}^{B G A}}{2}\left(\frac{V_{r}^{B G A^{\prime}} \rho_{2}}{1-\eta_{1}^{B G A}}\right)^{2} \\
& \left.+V_{m}^{B G A^{\prime}}\left(\frac{\rho_{1}^{2} V_{r}^{B G A^{\prime}}}{\eta_{2}^{B G A}}+\frac{\rho_{2}^{2} V_{r}^{B G A^{\prime}}}{1-\eta_{1}^{B G A}}-\delta R^{B G A}\right)\right\} .
\end{aligned}
$$


Taking the first-order conditions with respect to $p_{1}^{B G A}, \omega^{B G A}, \eta_{1}^{B G A}$ and $\eta_{2}^{B G A}$ to maximize the manufacturer, we obtain

$$
\begin{gathered}
p_{1}^{B G A}=\frac{N_{2} \sqrt{R^{B G A}}}{2\left(b^{2}-1\right)}, \\
\omega^{B G A}=\frac{N_{3} \sqrt{R^{B G A}}}{2 b\left(b^{2}-1\right)}, \\
\eta_{1}^{B G A}=\frac{2 V_{m}^{B G A^{\prime}}-V_{r}^{B G A^{\prime}}}{2 V_{m}^{B G A^{\prime}}+V_{r}^{B G A^{\prime}},} \\
\eta_{2}^{B G A}=\frac{V_{R}^{B G A^{\prime}}}{2 V_{m}^{B G A^{\prime}}+V_{r}^{B G A^{\prime}}}
\end{gathered}
$$

Then, substituting (80) and (81) into (76), we obtain

$$
p_{2}^{B G A}=\frac{N_{4} \sqrt{R^{B G A}}}{4 b\left(b^{2}-1\right)}
$$

We look for the linear value functions,

$$
\begin{aligned}
& V_{m}^{B G A}=h_{1} R^{B G A}+h_{2}, V_{m}^{B G A^{\prime}}=h_{1}, \\
& V_{r}^{B G A}=h_{3} R^{B G A}+h_{4}, V_{r}^{B G A^{\prime}}=h_{3} .
\end{aligned}
$$

where $h_{1}, h_{2}, h_{3}, h_{4}$ are the parameters. Substituting (77)-(78) and (80)-(84) into HJB equations and simultaneous equations (85) and (86), we can obtain:

$$
\begin{aligned}
4 b N_{2}\left(b^{2}-1\right) \theta & -2 b^{2} N_{2}^{2}+N_{2} N_{4}+4\left(b^{2}-1\right)(1-\theta) N_{3}-N_{3} N_{4}+2 N_{2} N_{3} \\
& +8 b\left(b^{2}-1\right)^{2} \Delta_{1}-4 b \lambda \Delta_{1} N_{2}\left(b^{2}-1\right)-2 \Delta_{1} \lambda N_{4}\left(b^{2}-1\right) \\
& =8 b\left(b^{2}-1\right)^{2}(r+\delta) h_{1} \\
-(1- & \left.\eta_{1}^{2}\right)^{2} h_{3}^{2} \rho_{1}^{2}\left(1-\eta_{2}\right)\left(1-\eta_{1}\right)-\eta_{2}^{2} \eta_{1} h_{3}^{2} \rho_{2}^{2}+2 \eta_{2}\left(1-\eta_{1}\right) h_{1} h_{3} \rho_{1}^{2} \\
& +2\left(1-\eta_{1}\right) \eta_{2}^{2} h_{1} h_{3} \rho_{2}^{2}=2 r h_{2} \eta_{2}^{2}\left(1-\eta_{1}^{2}\right)^{2} \\
& \\
4\left(b^{2}-1\right)( & \left.N_{4}-2 N_{3}\right)(1-\theta)-N_{4}\left(N_{4}-2 N_{3}\right)+2 N_{2}\left(N_{4}-2 N_{3}\right)+16 b\left(b^{2}-1\right)^{2} \Delta_{2} \\
& -8 b \Delta_{2} N_{2} \lambda\left(b^{2}-1\right)-4 \Delta_{2} N_{4} \lambda\left(b^{2}-1\right. \\
& =16 b\left(b^{2}-1\right)^{2}(r+\delta) h_{3} \\
h_{3}^{2} \rho_{2}^{2} \eta_{2} & +h_{3}^{2} \rho_{1}^{2}\left(1-\eta_{1}\right)=2 r h_{4} \eta_{2}\left(1-\eta_{1}\right) .
\end{aligned}
$$

simultaneous equations (87)-(90), we can easily obtain the parameters $h_{1}, h_{2}, h_{3}, h_{4}$. And inserting $h_{1}$ and $h_{3}$ into (77)-(78) and (82)-(83), respectively, we can easily get

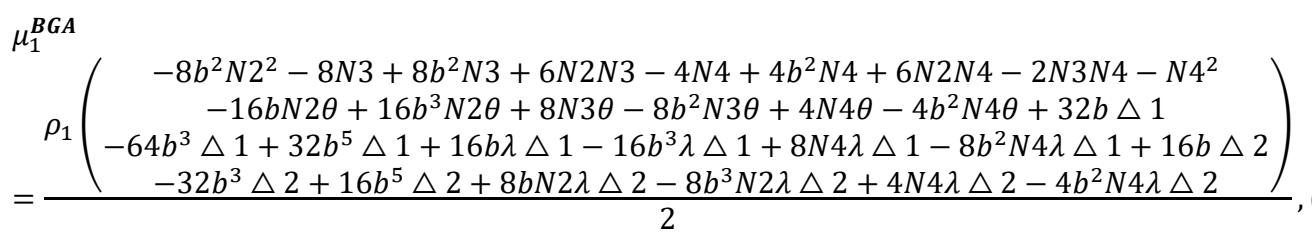




$$
\begin{aligned}
& \mu_{2}^{B G A} \\
& \rho_{2}\left(\begin{array}{c}
-8 b^{2} N 2^{2}-8 N 3+8 b^{2} N 3+6 N 2 N 3-4 N 4+4 b^{2} N 4+6 N 2 N 4-2 N 3 N 4-N 4^{2} \\
-16 b N 2 \theta+16 b^{3} N 2 \theta+8 N 3 \theta-8 b^{2} N 3 \theta+4 N 4 \theta-4 b^{2} N 4 \theta+32 b \triangle 1 \\
-64 b^{3} \triangle 1+32 b^{5} \triangle 1+16 b \lambda \triangle 1-16 b^{3} \lambda \triangle 1+8 N 4 \lambda \triangle 1-8 b^{2} N 4 \lambda \triangle 1+16 b \triangle 2 \\
-32 b^{3} \triangle 2+16 b^{5} \triangle 2+8 b N 2 \lambda \triangle 2-8 b^{3} N 2 \lambda \triangle 2+4 N 4 \lambda \triangle 2-4 b^{2} N 4 \lambda \triangle 2
\end{array}\right) \\
& =\frac{(}{2},
\end{aligned}
$$

$$
\begin{aligned}
& \eta_{1}^{B G A} \\
& =\frac{\left(\begin{array}{c}
-8 b^{2} N 2^{2}-24 N 3+24 b^{2} N 3+10 N 2 N 3+4 N 4-4 b^{2} N 4+2 N 2 N 4-6 N 3 N 4 \\
+N 4^{2}-16 b N 2 \theta+16 b^{3} N 2 \theta+24 N 3 \theta-24 b^{2} N 3 \theta-4 N 4 \theta+4 b^{2} N 4 \theta+32 b \triangle 1 \\
-64 b^{3} \triangle 1+32 b^{5} \triangle 1+16 b \lambda \triangle 1-16 b^{3} \lambda \triangle 1+8 N 4 \lambda \triangle 1-8 b^{2} N 4 \lambda \triangle 1-16 b \triangle 2 \\
+32 b^{3} \triangle 2-16 b^{5} \triangle 2-8 b N 2 \lambda \triangle 2+8 b^{3} N 2 \lambda \triangle 2-4 N 4 \lambda \triangle 2+4 b^{2} N 4 \lambda \triangle 2
\end{array}\right)}{\left(\begin{array}{c}
-8 b^{2} N 2^{2}-8 N 3+8 b^{2} N 3+6 N 2 N 3-4 N 4+4 b^{2} N 4+6 N 2 N 4-2 N 3 N 4-N 4^{2} \\
-16 b N 2 \theta+16 b^{3} N 2 \theta+8 N 3 \theta-8 b^{2} N 3 \theta+4 N 4 \theta-4 b^{2} N 4 \theta+32 b \triangle 1 \\
-64 b^{3} \triangle 1+32 b^{5} \triangle 1+16 b \lambda \triangle 1-16 b^{3} \lambda \triangle 1+8 N 4 \lambda \triangle 1-8 b^{2} N 4 \lambda \triangle 1+16 b \triangle 2 \\
-32 b^{3} \triangle 2+16 b^{5} \triangle 2+8 b N 2 \lambda \triangle 2-8 b^{3} N 2 \lambda \triangle 2+4 N 4 \lambda \triangle 2-4 b^{2} N 4 \lambda \triangle 2
\end{array}\right) .}
\end{aligned}
$$

$$
\begin{aligned}
& \eta_{2}^{B G A} \\
& =\frac{2\left(\begin{array}{c}
-8 N_{3}+8 b^{2} N_{3}+2 N_{2} N_{3}+4 N_{4}-4 b^{2} N 4-2 N_{2} N_{4}-2 N_{3} N_{4}+N_{4}^{2} \\
+8 N_{3} \theta-8 b^{2} N_{3} \theta-4 N_{4} \theta+4 b^{2} N_{4} \theta-16 b \triangle_{2}+32 b^{3} \triangle_{2} \\
+16 b^{5} \triangle_{2}+8 b N_{2} \lambda \triangle_{2}-8 b^{3} N_{2} \lambda \triangle_{2}+4 N_{4} \lambda \triangle_{2}-4 b^{2} N_{4} \lambda \triangle_{2}
\end{array}\right)}{\left(\begin{array}{c}
8 b^{2} N_{2}^{2}+8 N_{3}-8 b^{2} N_{3}-6 N_{2} N_{3}+4 N_{4}-4 b^{2} N_{4}-6 N_{2} N_{4}+2 N_{3} N_{4}+N_{4}^{2} \\
+16 b N_{2} \theta-16 b^{3} N_{2} \theta-8 N_{3} \theta+8 b^{2} N_{3} \theta-4 N_{4} \theta+4 b^{2} N_{4} \theta-32 b \triangle_{1} \\
+64 b^{3} \triangle_{1}-32 b^{5} \triangle_{1}-16 b \lambda \triangle_{1}+16 b^{3} \lambda \triangle_{1}-8 N_{4} \lambda \triangle_{1}+8 b^{2} N_{4} \lambda \triangle_{1}-16 b \triangle_{2} \\
+32 b^{3} \triangle_{2}-16 b^{5} \triangle_{2}-8 b N_{2} \lambda \triangle_{2}+8 b^{3} N_{2} \lambda \triangle_{2}-4 N_{4} \lambda \triangle_{2}+4 b^{2} N_{4} \lambda \triangle_{2}
\end{array}\right) .}
\end{aligned}
$$

\section{Acknowledgement}

This research was partially supported by a research grant of Korea Research Foundation of 2017 (No. NRF-2016R1A2B1015318) and the Ajou University research fund of 2017.

\section{References}

[1] W. K. Chiang, D. Chhajed and J. D. Hess, "Direct marketing, indirect profits: a strategic analysis of dualchannel supply chain design", Manag. Sci., vol. 49, (2003), pp. 1-20.

[2] R. Yan, Z. Pei and C. Myers, "Do channel members value the multiple-cooperation strategy?", Journal of Retail. Consum. Serv., vol. 30, (2016), pp. 84-95.

[3] F. Gao and X. Su, "Omnichannel service operations with online and offline self-order technologies", Manag. Sci., vol. 64, (2017), pp. 3469-3970.

[4] S. C. Bulmus, S. X. Zhu, R. Teunter, "Competition for cores in remanufacturing", Eur. J. Oper. Res., vol. 233, (2014), pp. 105-113.

[5] V. D. R. Guide, R. H. Teunter and L. N. Van Wassenhove, "Matching demand and supply to maximize profits from remanufacturing", Manuf. Serv. Oper. Manag., vol. 5, (2003), pp. 303-316.

[6] T. M. Choi, Y. Li and L. Xu, "Channel leadership, performance and coordination in closed loop supply chains", Int. J. Prod. Econ., vol. 146, (2013), pp. 371-380.

[7] R. Geyer, L. N. Van Wassanhove and A. Atasu, "The economics remanufacturing under limited component durability and finite product life cycles", Manag. Sci., vol. 53, (2007), pp. 88-100.

[8] J. Chen and C. Chang, "The co-opetitive strategy of a closed-loop supply chain with remanufacturing", Transp. Res. E Logist. Transp. Rev., vol. 48, (2012), pp. 387-400.

[9] C. H. Chuang, C. X. Wang and Y. Zhao, "Closed-loop supply chain models for a high-tech product under alternative reverse channel and collection cost structures", Int. J. Prod. Econ., vol. 156, (2014), pp. 108123.

[10] J. D. Shulman, A. T. Coughlan and R. C. Savaskan, "Optimal Reverse Channel Structure for Consumer Product Returns”, Mark. Sci., vol. 29, (2010), pp. 1071-1085.

[11] M. Huang, M. Song, L. H. Lee and W. K. Ching, "Analysis for strategy of closed-loop supply chain with dual recycling channel”, Int. J. Prod. Econ., vol. 144, (2013), pp. 510-520.

[12] L. G. Debo, L. B. Tokay and L. N. Van Wassenhove, "Market segmentation and product technology selection for remanufacturable products", Manag. Sci., vol. 51, (2005), pp. 1193-1205.

[13] C. Jiang, F. Xu and Z. Sheng, "Pricing strategy in a dual-channel and remanufacturing supply chain system", Int. J. Syst. Sci., vol. 41, (2010), pp. 909-921.

[14] P. Majumder and H. Groenevelt, "Competition in remanufacturing”, Prod. Oper. Manag., vol. 10, (2001), pp. 125-141. 
[15] P. Yi, M. Huang, L. Guo and T. Shi, "A retailer oriented closed-loop supply chain network design for end of life construction machinery remanufacturing", J. Clean. Prod., vol. 124, (2016), pp. 191-203.

[16] L. White and G. J. Lee, "Operational research and sustainable development: Tackling the social dimension”, Eur. J. Oper. Res., vol. 193, (2009), pp. 683-692.

[17] G. Ferrer and J. M. Swaminathan, "Managing new and remanufactured products", Manag. Sci., vol. 52, (2006), pp. 15-26.

[18] L. Lu, X. Qi and Z. Liu, “On the cooperation of recycling operations”, Eur. J. Oper. Res., vol. 233, (2014), pp. 349-358.

[19] P. De Giovanni, P. V. Reddy and G. Zaccour, "Incentive strategies for an optimal recovery program in a closed-loop supply chain”, Eur. J. Oper. Res., vol. 249, (2016), pp. 605-617.

[20] R. C. Savaskan, S. Bhattacharya and L. N. Van Wassenhove, "Closed loop supply chain models with product remanufacturing", Manag. Sci., vol. 50, (2004), pp. 239-252.

[21] S. Saha, S. P. Sarmah and I. Moon, "Dual channel closed-loop supply chain coordination with a rewarddriven remanufacturing policy", Int. J. Prod. Res., vol. 54, (2016), pp. 1503-1517.

[22] R. C. Savaskan and L. N. Van Wassenhove, "Reverse channel design: the case of competing retailers", Manag. Sci., vol. 52, (2006), pp. 1-14.

[23] M. E. Ferguson and L. B. Toktay, "The effect of competition on recovery strategies", Prod. Oper. Manag., vol. 15, (2006), pp. 351-368.

[24] P. De Giovanni, "Environmental collaboration in a closed-loop supply chain with a reverse revenue sharing contract", Ann. Oper. Res., vol. 220, (2014), pp. 135-157.

[25] P. De Giovanni, P. V. Reddy and G. Zaccour, "Incentive strategies for an optimal recovery program in a closed-loop supply chain”, Eur. J. Oper. Res., vol. 249, (2016), pp. 605-617.

[26] P. De Giovanni, "A joint maximization incentive in closed-loop supply chains with competing retailers: the case of spent-battery recycling”, Eur. J. Oper. Res., vol. 268, (2018), pp. 128-147.

[27] C. J. Corbett and R. C. Savaskan, "Contracting and coordination in closed-loop supply chains", In Business aspects of closed-loop supply chains: Exploring the issues, 1st ed.; Guide, V.D., Van Wassenhove, L., Pittsburgh, Eds.; Carnegie Mellon University Press: Pittsburgh, PA, ISBN: 93-113, 088748-402-6, vol. 2, (2003).

[28] K. Govindan and M. N. Popiuc, "Reverse supply chain coordination by revenue sharing contract: A case for the personal computers industry", Eur. J. Oper. Res., vol. 233, (2014), pp. 326-336.

[29] P. De Giovanni, "Closed-loop supply chain coordination through incentives with asymmetric information”, Ann. Oper. Res., vol. 253, no. 1, (2017), pp. 133-167.

[30] T. Xiao, D. Yang and H. Shen, "Coordinating a supply chain with a quality assurance policy via a revenuesharing contract", Int. J. Prod. Res., vol. 49, no. 1, (2011), pp. 99-120.

[31] G. Cachon and M. Lariviere, "Supply chain coordination with revenue sharing contracts: Strengths and limitations”, Manag. Sci., vol. 51, (2005), pp. 30-44.

[32] P. De Giovanni and M. Roselli, "Overcoming the drawbacks of a revenue-sharing contract through a support program", Ann. Oper. Res., vol. 196, (2012), pp. 201-222.

[33] X. Hong, L. Xu, P. Du and W. Wang, "Joint advertising, pricing and collection decisions in a closed-loop supply chain”, Int. J. Prod. Econ., vol. 167, (2015), pp. 12-22.

[34] J. Xie, L. Liang, L. Liu and P. Ieromonachou, "Coordination contracts of dual-channel with cooperation advertising in closed-loop supply chains", Int. J. Prod. Econ., vol. 183, (2017), pp. 528-538.

[35] A. Atasu, L. B. Toktay and L. N. Van Wassenhove, "How collection cost structure drives a manufacturer's reverse channel choice", Prod. Oper. Manag., vol. 22, (2013), pp. 1089-1102.

[36] M. Nerlove and K. J. Arrow, "Optimal advertising policy under dynamic conditions", Economica, vol. 39, (1962), pp. 129-142.

[37] R. Yan, P. Guo, J. Wang and N. Amrouche, "Product distribution and coordination strategies in a multichannel context", J. Retail. Consum. Serv., vol. 18, (2001), pp. 19-26.

[38] J. Zhang, J. Xie and B. Chen, "Cooperative advertising with bilateral participation”, Decis. Sci., vol. 44, (2012), pp. 193-203.

[39] B. Giri, A. Chakraborty and T. Maiti, "Pricing and return product collection decisions in a closed-loop supply chain with dual-channel in both forward and reverse logistics", J. Manuf. Syst., vol. 42, (2017), pp. 104-123.

[40] J. Song, F. Li, D. D. Wu, L. Liang and A. Dolgui, "Supply chain coordination through integration of innovation effort and advertising support", Appl. Math. Model., vol. 49, (2017), pp. 108-123. 